\title{
Cécile Cloutier
}

Université de Toronto

\section{L'Humanisme de Cendrars}

$\mathbf{L}$

ire Cendrars, c'est participer à une fête. En effet, pour lui, l'important, c'est de se sentir heureux. Son oeuvre est une somme de toutes les sensations, de tous les sentiments du monde. Sur toutes choses, il multiple les idées et les impressions. Homme de l'univers entier, it a vécu pour le chanter de sa voix nue. Plein d'énergie et de vitalité, il fut délicieusement sage et fou. De fait, toute sa vie fut une incarnation de la jeunesse. Il a parlé en homme du réel des importances à assumer et de la rassurante cohérence de la vie.

Il est toujours un peu bête de dire d'un être humain qu'il est complet. Et pourtant, on doit accorder à Cendrars d'avoir peut-être mérité cette importante définition. En effet il a voulu tout connaitre, tout aimer, tout vivre. D'une érudition encyclopédique, il a cherché à savoir ou à deviner tous les secrets des animaux, des plantes ou des pierres. Il doit aussi beaucoup à la fatigue, à la faim et à la misère. Il a aimé profondément tous les peuples dans leurs ressemblances et dans leurs différences. Il s'est intéressé passionnément à l'Amérique du Sud et surtout au Brésil. Il a été attiré par la civilisation des Noirs, poète de l'immédiat, il était fait pour la comprendre. Il adorait la façon dont elle s'approche tout près de la nature et garde contact avec les forces primitives. Par exemple, il croit que les Africains adorent le serpent qui touche à la terre de tout son corps et connait ainsi tous les secrets de l'univers. Rappelons-nous qu'il a publié une anthologie nègre. Tous ses voyages furent une recherche de l'Amour.

Il a aussi constamment reçu beaucoup de la simplicité de l'homme du peuple. Lui-même ne s'est jamais pris au sérieux. Né de père suisse et de mère écossaise, à quinze ans il se sauva de sa chambre du cinquième étage pour se laisser tomber de balcon en balcon. Ainsi finit sa vie bourgeoise. N'oublions pas non plus que 
son amour de l'aventure le conduisit au Canada où il travailla dans une ferme de l'Ouest.

Cendrars a donc toujours été un innovateur, un précurseur. En littérature, il s'est vraiment voulu la conscience de son époque. Pour lui, le vingtième siècle est celui du départ à zéro, du tragique et de la lucidité. Dans ce monde moderne qu'il veut nous montrer beau, il retrouve les grands thèmes éternels: l'amour, la mort, la misère, l'énergie et l'admiration. Il a compris le besoin d'activité de l'homme d'aujourd'hui et son immense désir d'autre chose. Dans un autre temps, Cendrars eut été Marco Polo. Il a senti profondément que maintenant "Un rond n'est plus un cercle mais devient une roue." La poésie, pour la première fois peut-être n'appartient à aucun mouvement mais s'éprend seulement de l'habitable. Il fut amoureux de la machine et du jazz auxquels il a emprunté plusieurs techniques de même qu'au cinéma dont il a vu l'aspect prophétique et dont il a senti qu'il pouvait faire des miracles. Ses livres procèdent très souvent par découpages cinématographiques. Il est alors surtout influencé par Griffith. Il a voulu adopter en particulier la vie de son temps et l'on peut rêver de ce qu'il aurait dit des voyages à la lune.

D'ailleurs l'espace prend dans sa vie et dans son oeuvre une place immense et intense. Notons seulement son goût d'habiter, d'avoir plusieurs lieux dans l'univers. Souvenons-nous qu'il eut à un certain moment de sa vie vingt-sept demeures sur la planète terre.

Mais cet univers des objets n'est au fond qu'un visage du monde de l'homme, lequel adopte tout au long de l'oeuvre de Cendrars les multiples aspects de la fraternité. Ne retenons que sa passion pour les trains qui nous a donné l'émouvant "Transibérien" ou ce culte pour l'Alfa-Romeo dessinée par Braque et qu'il conduisit avec tendresse d'une seule main jusqu'aux confins de l'Amérique du Sud où il rencontra ce merveilleux pompiste du Brésil, cet humaniste raffiné qui ne savait pas lire mais qui sculptait interminablement des statues du chemin de la croix dont tous les personnages étaient représentés en automobile.

Car c'est à cause des hommes qu'il s'intéresse aux objets. C'est pour lui une façon d'être profondément honnête envers eux. Il les considère comme capables d'éveiller les autres. L'univers lui apparaît comme un tout relié à l'humain. Cendrars cherche d'abord à établir la communication de tout à tout. Par exemple, il a toujours regretté 
de ne pouvoir partager sa joie à fond car il ne voulait pas s'épanouir seul. Toute sa vie, il a voulu être solidaire des autres et leur aider, trouvant toujours que c'est le rôle humain le plus difficile. Il a souvent constaté avec tristesse cette vérité que connaît toute mère en face d'un enfant malade ou malheureux: l'amour le plus grand ne peut pas tuer la souffrance.

Bien sûr, il a eu des amis merveilleux parce qu'il était lui-même un ami extraordinaire. Ne soulignons que cette amitié avec Charlie Chaplin, commencée alors qu'ils étaient tous les deux pauvres et inconnus à Londres et puis cette immense bienveillance pour Sawo, le prince des Gitans et pour toute sa tribu et puis Gustave le Rouge, et puis Eric Satie, et puis Apollinaire si bien cendrarisés que l'on ne sait plus s'ils étaient les amis de Galmot, de Suter, de Moravagine, de Dan Yack ou de Blaise Cendrars.

Quoique Cendrars n'ait jamais été l'homme d'un système, il a réfléchi sur le sens de la vie. D'ailleurs il croit que l'homme ne justifie rien sinon lui-même et qu'il doit marcher dans le sens de l'instant où il vit, mais alors, il doit atteindre le paroxysme. Cendrars a eu avant tout le sens du possible et il a voulu aller jusqu'au bout, réinventer chaque journée à chaque jour. C'est ainsi qu'il contredit l'absurdité de l'existence dont il fut douloureusement conscient. Il faut donc être ardent et rechercher le secret de tout. On doit vivre énergiquement, intensément le quotidien et faire comme si l'absurde n'existait pas. Mais le hasard régit tout et la destinée est implacable et contraire à l'homme. Seule l'action peut influencer le destin et d'elle, le bonheur peut naître. De cette façon, l'homme peut sérieusement s'opposer au désordre, mais l'indifférence demeure toujours l'une des formes de la sagesse et aussi l'une des puissances qui entrainent l'homme vers l'inconnu et celui-ci doit se tourner du côté de sa vraie nature. Pourtant, alors, quoique bouleversant tout, il reste seul. Et Cendrars fut un grand solitaire, mais il était convaincu qu'il faut être différent pour vivre. Cependant la contemplation fut toujours son essence même s'il était certain qu'elle est aussi une aventure. Mais cet homme de silence sut accorder sa vie intérieure à sa vie extérieure et créer ce qu'il aurait pu voir. En ce sens, il fut à la fois visionnaire et témoin, considérant la vie comme la seule chose absolument certaine. 
Il croit aussi dans la beauté du mouvement: “J'aurai été l'un des premiers poètes du temps à voulour mener ma vie sur un plan mondial." Mais l'action, ou plutôt l'acte demeure son seul moyen de libération. Il veut donc reconstruire le monde en agissant.

Cependant il fut aussi fasciné par une vérité qui est hors de ce monde. Il s'est intéressé à la mystique des Noirs, à la magie et à la Kabbale, et c'est par la fraternité qu'il est venu à l'art. Il devint une sorte de mystique laïc et il aurait pu être un prophète hippie. Dan Yack et Galmot seront avant tout des êtres d'amour et de charité qui chercheront constamment à aller au bout des choses. Ce seront des saints qui voudront un après et un plus loin. L'homme continuant d'avoir besoin de croire et la science et la psychanalyse ayant constitué une foi en quelque sorte passagère, les saints cendrariens seront des vivants en plein coeur du monde. Après s'être intéressé aux recherches sur l'inconscient, aux mystères des religions et à la surréalité, Cendrars retournera au réel. Pour lui, l'expérience sera donc une sorte de phénomène religieux et elle aura une valeur première.

Pourtant cette vie tant célébrée est aussi le germe de la mort. De fait, la condition humaine est désespérée. D'ailleurs, la mort montre l'absurdité du monde. C'est au moins l'une des seules réalités évidentes que l'homme possède. La vie n'est au fond qu'un itinéraire entre deux pôles et puisque tout le mouvement du monde est irréversible, la mort est peut-être un début. Mais, Cendrars refuse le suicide, la guerre et même la drogue. Il croit que l'envergure d'un homme s'évalue beaucoup selon la façon dont il pense à la mort et que, de toutes façons, le but final doit préoccuper moins que l'instant actuel.

Il faut donc vivre librement car la liberté contredit l'absurdité. Il était convaincu que l'action libère et ses personnages agissaient dans ce but. Dan Yack est peut-être en ce sens le héros le plus libre qu'il ait créé et en même temps le plus près de l'absolu. La glace le libère et le purifie. C'est un vrai héros et Cendrars est peut-être l'un des rares écrivains contemporains à avoir créé des héros. En effet, ses personnages ont une grandeur épique.

Il sait que l'unité de l'homme moderne existe mais qu'elle est profonde. Elle ne peut se permettre d'être une privation de la réalité. Cendrars disait: "Je ne veux pas de l'université mais de l'univers." 
L'unité est sous la pluralitê, au bout du total. Et Suter et Moravagine et Galmot vivent ce que Cendrars a appelé la simultanéité. Et ils restent disponibles tout en s'engageant.

Les personnages de Cendrars sont aussi conscients. Ils boivent peu et ne se droguent pas. "J'aime ma luciditê," dira Dan Yack. Ils sont à la fois, intelligents et sensibles, et si le dix-huitième s'est signalé comme le siècle de l'intelligence et le Romantisme du dixneuvième comme un moment de sensibilité, le vingtième a voulu réaliser cette synthèse magnifique d'un homme à la fois intelligent et sensible.

Le héros de Cendrars est, avant tout, un homme d'aujourd'hui. C'est un être libre qui refuse les rôles et les cadres, qui veut être lui-même le dramaturge de sa vie, qui cherche à la réinventer. C'est l'être des grandes soifs et des grandes faims. C'est un voyageur d'envergure géographique qui abolit les distances, et confond espace et temps. Il nous donne des images inoubliables de l'Afrique et de l'Amérique du Sud. S'il y a un humanisme nègre de Cendrars, il y a aussi une innocence de nouveau monde chez ses personnages.

Mais ce monde moderne avec ses problèmes modernes, Cendrars le rattache à la tradition. Et lui et ses personnages sont des gens qui savent. Cendrars était d'une culture phénoménale. Il avait tout lu, même l'horaire des chemins de fer français. Ses mains, et plus tard, son unique main, savalent tous les métiers.

Ses héros sont à son image. Ils essaient avant tout d'être bienfaisants pour les autres, sur le plan social, politique, quotidien, tout simplement humain. Ce sont des hommes justes et sages. Il faut savoir prendre les gens comme ils sont et les mensonges font aussi partie de la personnalité. Les héros de Cendrars aiment et luttent. Il y a des procès dans Cendrars. Souvenons-nous de Galmot. Cendrars écrivait d'ailleurs que "gagner de l'argent est intéressant parce que c'est une lutte."

Les personnages de Cendrars sont des versions modernes des héros médiévaux. Ils ont des grandeurs épiques. Ce sont aussi des phénomènes de bonne santé. Ils entreprennent des tâches surhumaines et vivent en même temps plusieurs vies humaines. Modernes, les héros de Cendrars le sont encore par l'attention donnée aux nombres. Ceux-ci ont une valeur sacrée. Ces personnages vivent 
dans un monde habité de chiffres. Ceux-ci ont une fonction de signes et ils sont importants pour les hommes.

Le personnage de Cendrars a cependant un trait par lequel il n'est pas conventionnellement moderne. Il ne se sent pas condamné comme celui de Camus, de Sartre ou de Malraux. Il est innocent. Il ne lutte pas contre des névroses mais contre des êtres et des objets. Aux lecteurs, Cendrars demande d'être les citoyens d'un monde plein de santé, où Dieu existe et où, à force de courage et d'amour on peut être heureux. Car, pour lui, les saints restent des exceptions.

Mais au-delà de toutes ces considérations, la réalité profonde des personnages de Cendrars consiste en la sensation. La terre existe chaudement et elle est bonne. Ils l'apprécient de tous leurs sens. Ils recherchent sans cesse le visible, l'audible, le palpable et l'olfactif. Moravagine dira: "Je reconnais tous les pays, les yeux fermés, à leur odeur.” Pour Cendrars, la Méditerranée sent l'armoire à linge et la confiture. Il invente le monde par les sens. Tout est sapide, tangible. Et c'est ainsi qu'il nous apprend l'homme, la bête, la plante, l'astre et la pierre. Ses livres sont bons comme le pain. Pour les comprendre, il faut faire plus que de l'exégèse critique, il faut aimer les abeilles et tout ce qui se passe de simple et de merveilleux dans le monde.

Il réhabilite les choses qui au vingtième siècle ont pris tellement d'importance à cause de la science et aussi parce que depuis des siècles, l'homme est déçu par l'homme. Ces objets que, à cause de leurs humbles et patients travaux, les hommes n'avaient jamais oubliés mais abstraits, Cendrars les écoute et il en apprend des leçons. L'automobile, par exemple, est pour lui un prolongement de l'homme. Elle le multiplie. Ce monde des objets, il l'atteint avant tout, par le travail, une autre réalité mise tellement à la mode au vingtième siècle. Il le transforme amicalement, chaudement, par la bonté de ses deux mains.

Mais si Cendrars fut un homo faber, il fut aussi un homo loquens. Il a voulu transcendre l'absurdité par l'écriture littéraire qui est pour lui une survivance. En quelque sorte, elle va plus loin que l'art. Il veut découvrir le secret de l'homme et du monde. Le langage jaillit du plus profond et va vers le plus profond et écrire est une façon de fuir le néant et de découvrir de nouveaux bonheurs.

Ses livres sont aussi l'histoire d'un dépouillement au bout duquel on arrive à la vie. Il s'en veut le célébrant et le visionnaire et 
il désire que sa parole soit pure richesse et enchantement tout en demeurant salvatrice. Il veut libérer le présent et son oeuvre est tout simplement le témoignage de ce que l'on vit sans recherche. D'ailleurs, le vivre ne doit pas être parfait, achevé. Plutôt, il revient au lecteur de le continuer. En ce sens, il renouvelle complètement la vision poétique de son temps. Par exemple, il met l'imaginaire dans le réel et il écrit: "Je ne trempe pas ma plume dans un encrier mais dans la vie." On peut dire qu'il a pillé le monde entier pour faire ses livres. D'ailleurs, pour luí, écrire un poème était un luxe, il fallait le vivre.

Et c'est par cette parole revenue à l'origine et retournée vers l'autre qu'il affirme la grandeur de l'homme et des bêtes et qu'il accorde sa joie à celle de la nature. L'écriture lui fut tout simplement ce par quoi on parle des réalités qui existent. Il demeure donc un écrivain total.

Il n'y a pas de salut pour lui sinon au milieu des hommes et de l'histoire. Cendrars pensait que la légende était dans l'actualité, dans le nouveau. Il considérait l'oeuvre littéraire comme un reflet du monde. Ses livres sont une manière de vivre et non une écriture.

Les personnages de Cendrars sont intéressés par le spectacle du monde et par sa représentation. Et la représentation n'est pas que l'oeuvre d'art dans le sens traditionnel. Pour Cendrars, celui qui monte une entreprise commerciale, représente le monde à sa façon: il crèe.

Vivre est un art magique. Celui qui vit doit transformer. Et les personnages de Cendrars sont avant tout des vivants et cela est rare. Il faut vivre "sans virgule" disait Cendrars. La vie est dangereuse et celui qui agit doit aller jusqu'au bout de son acte sans se plaindre. Pour un personnage de Cendrars, le destin n'est rien d'autre que la vigueur. Il est à remarquer que ses personnages n'écrivent pas. L'oeuvre de leur vie, c'est de vivre.

Mais pour lui, Cendrars, écrire est un acte vital. C'est brûler vif. Il a aimé passionnément l'aujourd'hui. A toute littérature, il a préféré l'actuel. Il a écrit du mouvement, de la dynamique. Jamais peut-être l'écriture n'avait autant dépassé l'écriture.

Et pourtant, cet écrivain de l'expérience demeure aussi celui de la simplicité. Il ne pense pas à faire une oeuvre, elle jaillit dans la générosité. D’ailleurs, écrire est beaucoup plus créer des person- 
nages et des situations que leur donner une forme. Il pensait qu'on ne peut juger sérieusement d'un écrivain en ne tenant compte que de son style. Tout dans son oeuvre va donc correspondre à une manière d'être. Proportions et angles seront changés et tout pourra devenir sujet de littérature, tout pourra exister pour le langage. Il écrira d'une façon goulue et avec une franchise totale. Sa poésie sera intense réalisant une fusion presque parfaite entre l'écrivain et son écriture.

Il voit que nous vivons un nouveau Moyen Age, et il veut désintellectualiser le livre. L'écriture devient une forme de révolte et elle fuit la littérature. Il se sent prisonnier de la parole et il cherche à éloigner l'art de l'esthétique. Il a aussi compris que le livre de demain sera le livre et non telle catégorie de livre.

Il voudra encore que l'oeuvre naisse d'une longue et belle patience. Il pourra mettre des années à faire un bouquin, le nourrissant de toute la sagesse que chaque jour lui apporte. Puis, au moment où il l'écrira, il doutera de sa perfection artistique. L'incubation sera très longue et il détestera l'horaire et l'échéance répétant qu'il tient à être d'abord homme et écrivain ensuite. Est-ce la raison pour laquelle il renoncera si tôt à écrire de la poésie?

Pourtant, il fut un éternel amoureux du vocabulaire et il s’intéressa beaucoup à la technique littéraire. L'un des premiers, il voulut écrire comme on parle. Il se passionna aussi pour la chanson. De l'affiche, il retint des effets de surprise, d'accumulation et d'effervescence. Il adorait la métaphore qu'il cherchait à construire en évitant l'intelligence, en faisant confiance à l'instinct.

Il s'intéressa aussi énormément à la peinture et la seule école artistique à laquelle il consentit peut-être fut le simultanéisme de Robert Delaunay. Cendrars, comme le peintre, avait toujours adoré la couleur dans le monde et le simultanéisme voulait redonner la sensation comme elle est. Cendrars, bouleversé par cette nouvelle façon de voir l'art, alla vivre chez Delaunay à son retour d'Amérique en 1912. Il adopta le simultanéisme en littérature, convaincu qu'à un certain niveau, les événements ne se succèdent pas mais sont simultanés. Il découvrit aussi que le contraste est plus profondément une ressemblance et que, par exemple, lamour est basé sur lui. Cendrars va donc, comme Delaunay, centrer son art sur le monde extérieur et comme lui, considérer la lumière comme une forme et 
non comme une essence. Il fera alors une édition de poésie simultanée "Premier livre simultané," un dépliant de deux mètres de hauteur illustré avec des couleurs de Sonia Delaunay. Il dira à l'occasion de ce bouquin: "Il ne s'agit plus de vision simultanée en art, ce qui a toujours existé; il s'agit du nouveau métier représentatif et simultané: peinture, sculpture, ameublement, architecture etc. etc." Mais, il refusera là encore que l'on s'enferme dans des principes. Cendrars a donc su voir l'inattendu et l'exceptionnel dans le monde, non par recherche mais par lucidité et intuition. Son univers insolite est authentique et quotidien. Et tout ce disparate est unifié par l'éclatante présence de l'humain. 